\title{
Weichteilsarkome des Erwachsenen
}




\title{
Beiträge zur Onkologie Contributions to Oncology
}

\section{Vol. 56}

\author{
Reihenherausgeber W. Queißer, Mannheim
}

W. Scheithauer, Wien 


\title{
Weichteilsarkome des Erwachsenen
}

Standards, Molekularbiologie, Perspektiven

\author{
Bandherausgeber F. W. Rath, Halle/Saale \\ M. Schönfelder, Leipzig
}

KARGER $\begin{aligned} & \text { Basel } \cdot \text { Freiburg } \cdot \text { Paris } \cdot \text { London } \cdot \text { New York } \\ & \text { New Delhi } \cdot \text { Bangkok } \cdot \text { Singapore } \cdot \text { Tokyo } \cdot \text { Sydney }\end{aligned}$ 


\section{Beiträge zur Onkologie Contributions to Oncology}

Die Deutsche Bibliothek - CIP-Einheitsaufnahme

Weichteilsarkome des Erwachsenen : Standards, Molekularbiologie, Perspektiven / Hrsg.: F.-W. Rath ; M. Schönfelder. - Basel ; Freiburg

[Breisgau] ; Paris ; London ; New York ; New Delhi ; Bangkok ; Singapore ;

Tokyo ; Sydney : Karger, 2002

(Beiträge zur Onkologie ; Bd. 56)

0101 deutsche buecherei

ISBN 3-8055-7394-4

Dosierungsangaben von Medikamenten

Autoren und Herausgeber haben alle Anstrengungen unternommen, um sicherzustellen, dass die Auswahl und Dosierungsangaben von Medikamenten im vorliegenden Text mit den aktuellen Vorschriften und der Praxis übereinstimmen. Trotzdem muss der Leser im Hinblick auf den Stand der Forschung, Änderung staatlicher Gesetzgebungen und den ununterbrochenen Strom neuer Forschungsergebnisse bezüglich Wirkung und Nebenwirkungen von Medikamenten darauf aufmerksam gemacht werden, dass unbedingt bei jedem Medikament die Packungsbeilage konsultiert werden muss, um mögliche Änderungen im Hinblick auf die Indikation und Dosierung nicht zu übersehen. Gleiches gilt für spezielle Warnungen und Vorsichtsmaßnahmen. Ganz besonders gilt dieser Hinweis für empfohlene neue und/oder nur selten gebrauchte Wirkstoffe.

Alle Rechte vorbehalten.

Ohne schriftliche Genehmigung des Verlags dürfen diese Publikation oder Teile daraus nicht in andere Sprachen übersetzt oder in irgendeiner anderen Form mit mechanischen oder elektronischen Mitteln (einschließlich Fotokopie, Tonaufnahme und Mikrokopie) reproduziert oder auf einem Datenträger oder einem Computersystem gespeichert werden.

(C) Copyright 2002 by

S. Karger GmbH, Postfach, D-79095 Freiburg, und

S. Karger AG, Postfach, CH-4009 Basel

Printed in Germany on acid-free paper by druckhaus köthen $\mathrm{GmbH}$

ISBN 3-8055-7394-4 


\section{Inhalt}

Geleitwort

Ch. Herfarth...................... XI

Vorwort

M. Schönfelder. . . . . . . . . . . . . . . . . . . XIII

\section{Allgemeine und klinische Pathologie}

F.-W. Rath, H.-J. Holzhausen, D. Kügler . . . . . . . . . . . . . 1

1.1 Definition und Klassifikation der Weichteilsarkome . . . . . . . . . 2

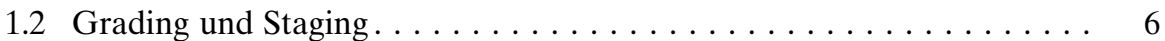

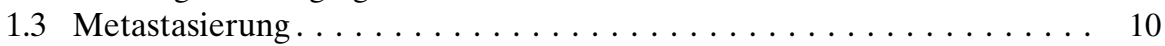

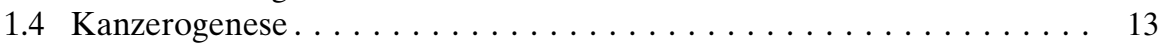

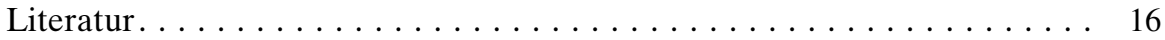

\section{Klinik, Diagnostik und Therapie}

P. Würl, M. Schönfelder. . . . . . . . . . . . . . . . . . . . . 19

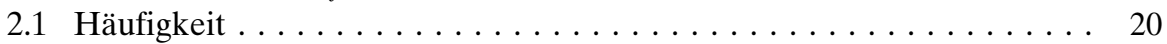

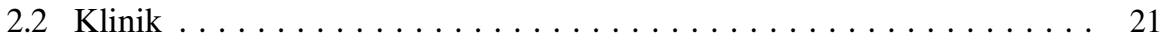

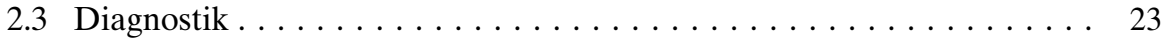

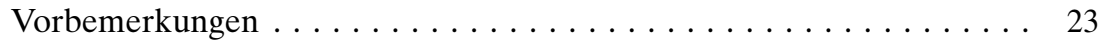

Bildgebende Diagnostik und Staging . . . . . . . . . . . . . . . 24

Labordiagnostik . . . . . . . . . . . . . . . . . . 25

Materialgewinnung zur prätherapeutischen histologischen

Untersuchung ...................... 27

2.4 Therapie . . . . . . . . . . . . . . . . . . . . . . . 29

Grundlegende chirurgische Therapieprinzipien . . . . . . . . . . . 29

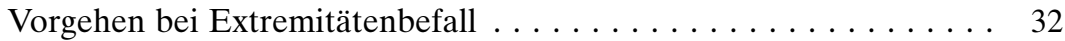

Besonderheiten an Thorax- und Bauchwand . . . . . . . . . . 33

Weichteilsarkome der Kopf- und Halsregion . . . . . . . . . . . . . . . 34 
Retroperitoneale und intraabdominale Sarkome . . . . . . . . . . . . 34

Vorgehensweise nach Enukleation oder Exzisionsbiopsie . . . . . . . . 36

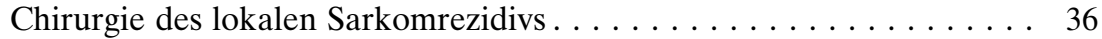

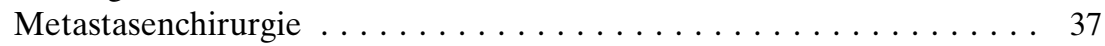

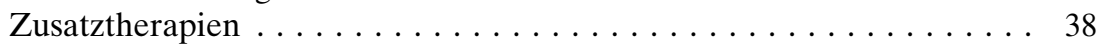

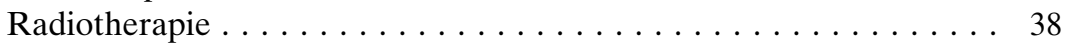

Chemotherapie . . . . . . . . . . . . . . . . . . . . 39

2.5 Prognose und konventionelle Prognosekriterien . . . . . . . . . . . . 42

Literatur . . . . . . . . . . . . . . . . . . . . . . . . . . . . 45

3 Molekulare Biologie . . . . . . . . . . . . . . . . . . . 51

3.1 Zytogenetik

H. Schmidt, R. Hinze. . . . . . . . . . . . . . . . . . . 52

Fibröse Tumoren. . . . . . . . . . . . . . . . . . 54

Fibrohistiozytische Tumoren $\ldots \ldots \ldots \ldots \ldots \ldots \ldots \ldots$

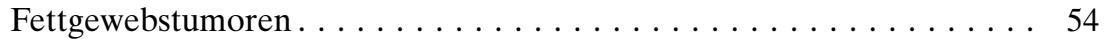

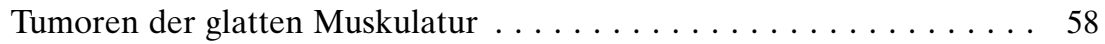

Tumoren der quergestreiften Muskulatur $\ldots \ldots \ldots \ldots \ldots \ldots$

Tumoren der Blut- und Lymphgefäße . . . . . . . . . . . . . . . . . 59

Perivaskuläre Tumoren . . . . . . . . . . . . . . . . . . . . 59

Mesotheliale Tumoren. . . . . . . . . . . . . . . . . . . . . . . 59

Neurale Tumoren . . . . . . . . . . . . . . . . . . . . . . . . . . 59

Extraskelettale Knorpel- und Knochentumoren. . . . . . . . . . . . 61

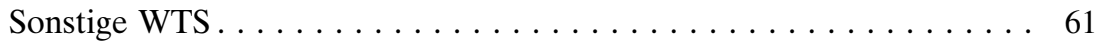

3.2 Angiogenese

J. Lasch . . . . . . . . . . . . . . . . . . . . . . . . . . . . 66

Allgemeine Vorbemerkungen. . . . . . . . . . . . . . . . 66

Aktueller Stand der Forschung. . . . . . . . . . . . . . . . . . . . . . 69

Perspektiven....................... 71

3.3 Onkogene und Tumorsuppressorgene

H. Taubert ........................... 71

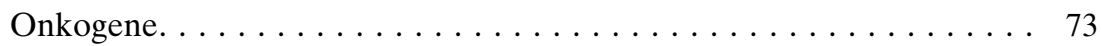

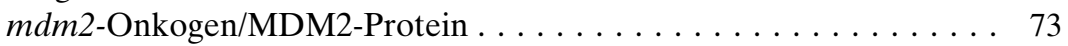

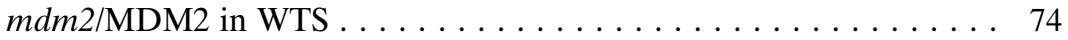

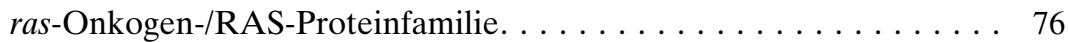

ras/RAS in WTS . . . . . . . . . . . . . . . . . . . 77

myc-Gen-/MYC-Proteinfamilie . . . . . . . . . . . . . . . 79

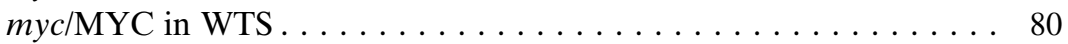

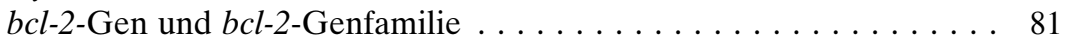

$b c l-2-G e n f a m i l i e$ in WTS $\ldots \ldots \ldots \ldots \ldots \ldots \ldots \ldots \ldots$

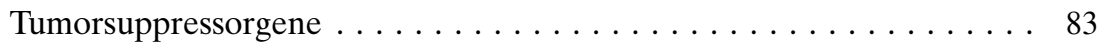

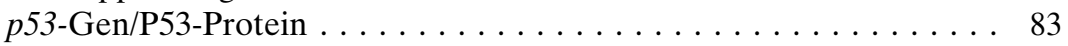

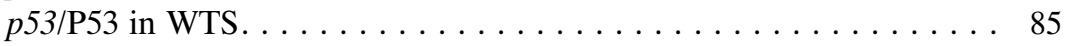

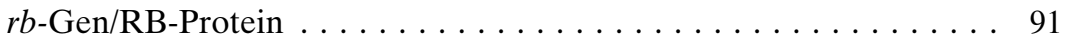

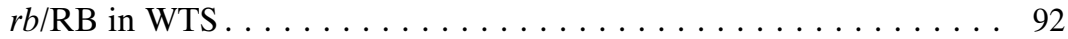


3.4 Apoptose

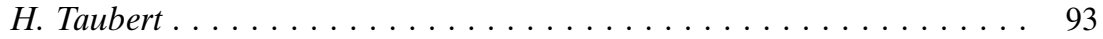

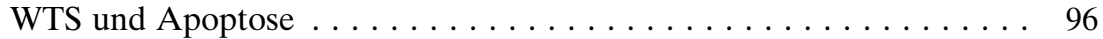

p53 und Apoptose . . . . . . . . . . . . . . . . . . . . . 97

FAS und Apoptose . . . . . . . . . . . . . . . . . . . 98

RB und Apoptose. . . . . . . . . . . . . . . . . . . . . 98

bcl-2-Genfamilie und Apoptose . . . . . . . . . . . . . . . . . 99

3.5 Zellzyklusregulierung und natürliche Wachstumskontrolle

A. Meye. . . . . . . . . . . . . . . . . . . . . . . 100

Zellzyklusregulierung . . . . . . . . . . . . . . . . 100

Alterationen von Zellzyklusregulatoren . . . . . . . . . . . . . 103

3.6 DNA-Reparatur und genomische Instabilität

A. Meye, H. Taubert . . . . . . . . . . . . . . . . . . . . . . . 111

DNA-Schädigung und Mechanismen der DNA-Reparatur . . . . . . . . 111

Mikrosatellitenstabilität - Defekte in DNA-Reparaturgenen . . . . . . 115

3.7 Strahleninduzierte Weichteilsarkome

H. Taubert . . . . . . . . . . . . . . . . . . . . . . 117

Literatur. . . . . . . . . . . . . . . . . . . . . . . . . . . . . 119

4 Neue tumortherapeutische Ansätze und ihre klinische Bedeutung . . 131

4.1 Nichtvirale und virale Gentherapie: Techniken und Ansätze

F. Bartel, J. Lasch . . . . . . . . . . . . . . . . . . . . . . . . . . . . 132

Nichtvirale Gentherapie . . . . . . . . . . . . . . . . . . . 132

Ziele der nichtviralen Gentherapie . . . . . . . . . . . . . . 133

Vehikel der nichtviralen Transfektionen . . . . . . . . . . . . . . . . . 134

Hauptbarriere für die Expression exogener DNA . . . . . . . . . . . 135

Versuche, die Effizienz des Kerntransports exogener DNA zu

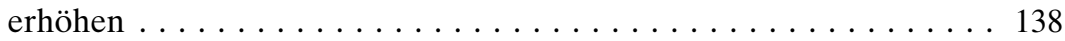

Was können wir von viralen Strategien, ihre DNA oder RNA in den

Wirtskern zu transportieren, lernen? . . . . . . . . . . . . . . . 139

Virale Vektorsysteme . . . . . . . . . . . . . . . . . . . . . . . . . 139

Adenoviren. . . . . . . . . . . . . . . . . . . . . . . 142

Retroviren . . . . . . . . . . . . . . . . . . . . . . 148

Lentiviren. . . . . . . . . . . . . . . . . . . . . 153

Therapieansätze für die Zukunft . . . . . . . . . . . . . . . . . 154

4.2 Bedeutung des Tumorsuppressorgens $p 53$ für die Entwicklung neuer

Therapiestrategien

P. Würl, H. Taubert. . . . . . . . . . . . . . . . . . . . . 155

In-vitro-Gentherapie. . . . . . . . . . . . . . . . . . 160

In-vivo-Ergebnisse an transgenen Mäusen und Ratten . . . . . . . . . . . 162

Klinische Phase-I-, -II- und -III-Studien . . . . . . . . . . . . . . . 163

Möglichkeiten zur Optimierung einer p53-Gentherapie . . . . . . . . . 165

4.3 Bedeutung von Arzneimittelresistenzgenen für klinische

Gentherapiestudien

P. Würl, J. Lasch. . . . . . . . . . . . . . . . . . . . . . . . . . . . 166 
4.4 Bedeutung des Onkogens $m d m 2$ für die Entwicklung neuer Therapiestrategien

A. Meye, F. Bartel . . . . . . . . . . . . . . . . . . . . . . . . 168

In-vitro-Ergebnisse . . . . . . . . . . . . . . . . . . . . . . . . 169

In-vivo-Ergebnisse . . . . . . . . . . . . . . . . . . . 173

Erste gentherapeutische Ansätze für WTS in vivo:

ein Ausblick . . . . . . . . . . . . . . . . . . . . . 176

4.5 Tumorangiogenese - ein möglicher gentherapeutischer Ansatzpunkt

J. Lasch, P. Würl . . . . . . . . . . . . . . . . . . . . . . . . . . . . . . . . 177

Strategien der gentherapeutischen Antiangiogenese. . . . . . . . . . . 177

Bedeutung der Angiogenesefaktoren für klinische Gentherapiestudien 178

Literatur . . . . . . . . . . . . . . . . . . . . . . . . . . . . . 181

\section{Schlussfolgerungen und Ausblick}

P. Würl.

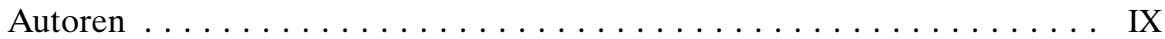

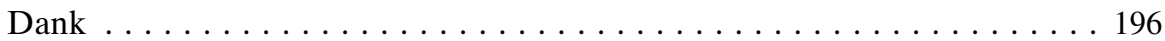

Glossar . . . . . . . . . . . . . . . . . . . . . . . . . . . 197

Sachwortverzeichnis . . . . . . . . . . . . . . . . . . . . . 201 


\section{Autoren}

Dipl.-Biochem. Frank Bartel

Dr. med. Raoul Hinze

Prof. med. Dr. Hans-Jürgen Holzhausen

Dr. med. Daniel Kügler

Prof. Dr. med. Friedrich-Wilhelm Rath

Dr. rer. nat. Hannelore Schmidt

PD Dr. habil. rer. nat. Helge Taubert

Institut für Pathologie

Martin-Luther-Universität Halle-Wittenberg

Magdeburger Straße 14

D-06097 Halle/Saale

Prof. Dr. med. Jürgen Lasch

Institut für Physiologische Chemie

Martin-Luther-Universität Halle-Wittenberg

Hollystraße 1

D-06097 Halle/Saale

Dr. rer nat. Axel Meye

Klinik für Urologie

Technische Universität Dresden

Fetscherstraße 74

D-01307 Dresden 
Prof. Dr. med. Manfred Schönfelder

Klinik und Poliklinik für Chirurgie I

Universität Leipzig

Liebigstraße 20 a

D-04103 Leipzig

PD Dr. med. Peter Würl

Chirurgische Klinik I

Universitätsklinikum Ulm

Steinhövelstraße 1

D-89075 Ulm 


\section{Geleitwort}

Ein mit Begeisterung geschriebenes Buch zur Diagnostik und Therapie der Weichteilsarkome liegt vor. Herausgeber und Autoren sind Kliniker und Grundlagenwissenschaftler. Schon dieses Konstrukt des beispielhaften Erfahrungs- und Wissenstransfers von Grundlagenwissenschaftlern in die Klinik ist zu loben. Darüber hinaus sind beide Gruppen - Kliniker wie Molekularbiologen - anerkannte und beachtete Vertreter ihres Fachs. Die Vereinigung beider Sichtarten dokumentiert gleichzeitig die Notwendigkeiten moderner klinischer Forschung mit direkter Verbindung zur Grundlagenwissenschaft.

Der molekularbiologische Teil des Werks gibt die ausgezeichneten Arbeiten der Gruppe über $p 53$ und MDM2 wieder. Auch die Untersuchungen zum Zellzyklus überzeugen. Allerdings sollten bei Weiterentwicklung und Anwachsen dieses Forschungsverbundes dann die wichtigen Fusionstranskripte mit eingebracht werden. Letztere gewinnen zunehmend an Bedeutung für die diagnostische Klassifizierung und therapeutische Folgerung. Natürlich wird jeder Optimismus für die Gentherapie begrüßt, und es befinden sich auch sehr interessante Ideen und vielversprechende Vektoren in Diskussion. Betrachtet man die Situation nüchtern, so muss doch noch mit einer Wartezeit von 5-10 Jahren gerechnet werden. Auf keinen Fall kann die entscheidende Rolle der chirurgischen Therapie mit dem Prinzip der kurativen Operation in den Hintergrund treten und der Eindruck entstehen, dass der Weg jetzt frei für eine «schlanke Chirurgie» wird, da potentielle molekulare Therapiemaßnahmen zur Verfügung stehen. Als Zielvision jedoch ist dies richtig.

Die Entwicklung geht weiter. Die großen klinischen Herausforderungen liegen in der diagnostischen und damit dann folgenden therapeutischen Einordnung der Weichteilsarkome, trotz ihrer erheblichen Unterschiedlichkeit. Die Inhomogenität lässt sich auf molekularem Weg besser zerlegen und definieren, um mögli- 
che neue therapeutische Ansätze einzuführen. Das große Verdienst des Werkes bleibt die Einbettung molekularbiologischer Forschung und Zusammenhangsanalysen in ein großes, gut gepflegtes und geführtes Krankengut. Ein Schritt ist getan. Weitere Wesentliche werden folgen.

Das Buch bestätigt den fruchtbaren Ansatz des in neuester Zeit propagierten grundlagenwissenschaftlichen-chirurgisch-klinischen Tandem-Modells

Prof. Dr. Dr. h.c. Ch. Herfarth, Heidelberg 


\section{Vorwort}

Die Entwicklung maligner Weichteiltumoren ist außerordentlich komplex, und sie tangiert noch unbekannte Basisphänomene des Lebens. Deshalb gibt es zur Zeit keine Möglichkeiten, das biologische Verhalten dieser Tumoren exakt zu ermitteln, weil nicht nur die Komplexität der Tumorentwicklung an sich, sondern ebenso die Wechselwirkung mit dem Tumorträger - also dem Patienten Analysen erheblich komplizieren.

Wenn auch noch viele biologische Abläufe eine Terra incognita sind, so werden durch molekularbiologische Erkenntnisse Reaktionsabläufe in den Tumorzellen, im Tumor selbst und in der Wechselbeziehung Tumor - Wirt zunehmend erkennbar. Diese molekularbiologischen Erkenntnisse eröffnen die Möglichkeit der therapeutischen Einflussnahme auf den Ablauf der Geschwulstkrankheit. So werden z. B. Interleukin 1 und 2, $\alpha$-Interferon, koloniestimulierende Wachstumsfaktoren wie die leukozyten- und makrophagenstimulierenden Wachstumsfaktoren sowie verschiedene molekulare Antikörper bereits klinisch genutzt oder in klinischen Studien auf ihre Wertigkeit und Verwendbarkeit überprüft. Auch therapeutische Genmanipulationen wurden durch neue Kenntnisse und technische Neuerungen ermöglicht. Es ist erkennbar, dass von verschiedenen Seiten Tumorgenese, Tumorwachstum und tumorbiologisches Verhalten molekularbiologisch untersucht werden und der Erkenntnisgewinn zur praktischen Überprüfung ansteht. Diese Entwicklung führte zu zunehmenden Basis- und Anwendungsstudien auf dem Gebiet der Krebsvakzination und der Gentherapie. Die bisherigen Resultate sind ermutigend. Die biologische Geschwulsttherapie verspricht neben zu erwartenden guten Erfolgen auch geringere Nebenwirkungen und Folgeschäden als die bisherige Chemotherapie. Deshalb wird sie mit wachsendem Interesse von Klinikern, aber auch von Nichtklinikern verfolgt.

Die vorliegende Monografie hat sich das Ziel gesetzt, unter Berücksichtigung der wesentlichsten neuen Erkenntnisse der molekularbiologischen For- 
schung, zugleich auch unter Verwendung eigener Forschungsergebnisse, Möglichkeiten der Gentherapie der malignen Weichteiltumoren darzustellen und zum Verständnis des tumorbiologischen Verhaltens dieser Tumorgruppe beizutragen. Die klinische und histopathologische Inhomogenität der Weichteilsarkome induziert geradezu den Wunsch, anhand molekularbiologischer Parameter Erklärungen für diese Heterogenität zu finden und eventuell einen gemeinsamen Nenner ihres tumorbiologischen Verhaltens, d. h. unabhängige Prognosefaktoren, zu eruieren.

Wegen des seltenen Auftretens von Weichgewebssarkomen werden in dem Buch eingangs die konventionelle Diagnostik und Therapie dargestellt, um dann auf der Basis molekularbiologischer Erkenntnisse auf adjuvante Therapiemöglichkeiten und tumorbiologische Reaktionsabläufe $\mathrm{zu}$ verweisen. Deshalb und weil die Vertreter beider Fachgebiete zukünftig gemeinsam diagnostisch und therapeutisch zusammenarbeiten müssen, wurde diese Monografie sowohl von Klinikern als auch von Basiswissenschaftlern verfasst. Wir hoffen, mit dieser interdisziplinären Arbeit beide Tätigkeitsfelder anzusprechen und das Bewusstsein für die Notwendigkeit einer intensiven Kooperation zu festigen.

Prof. Dr. M. Schönfelder, Leipzig 